\title{
Emittance Formula for Slits and Pepper-pot Measurement
}

\author{
Min Zhang \\ Fermi National Accelerator Laboratory \\ P.O. Box 500, Batavia, Illinois 60510
}

October 1996 


\section{Disclaimer}

This report was prepared as an account of work sponsored by an agency of the United States Government. Neither the United States Government nor any agency thereof, nor any of their employees, makes any. warranty, express or implied, or assumes any legal liability or responsibility for the accuracy, completeness or usefulness of any information, apparatus, product or process disclosed, or represents that its use would not infringe privately owned rights. Reference herein to any specific commercial product, process or service by trade name, trademark, manufacturer or otherwise, does not necessarily constitute or imply its endorsement, recommendation or favoring by the United States Government or any agency thereof. The views and opinions of authors expressed herein do not necessarily state or reflect those of the United States Government or any agency thereof.

\section{Distribution}

Approved for public release: further dissemination unlimited. 


\title{
Emittance Formula for Slits and Pepper-pot Measurement
}

\author{
Min Zhang $^{1}$
}

\section{Abstract}

In this note, a rigid formula for slits and pepper-pot emittance measurement is derived. The derivation is based on the one-dimensional slit measurement setup. A mathematical generalization of the slit emittance formula to the pepper-pot measurement is discussed.

\section{Introduction}

With the development of high precision linear accelerator technologies, there comes a strong demand for high quality beam measurements. For example, in a Self-Amplified Spontaneous Emission (SASE) free electron laser [1], it is required that the beam have as low as a few $\pi \mathrm{mm}$ mrad normalized transverse emittance at a $\mathrm{GeV}$ energy level. For such small emittances, a precise measurement is badly needed.

Two commonly used emittance measurement methods are: slits and pepper-pot (Fig. 1). The former is a one-dimensional emittance measurement device and the latter a two-dimensional one. For a space-charge dominated beam, the pepper-pot setup is more appropriate. It cuts an incident beam into small pieces - beamlets, which each has a very low charge. Figure 2 shows a typical setup of such measurements.
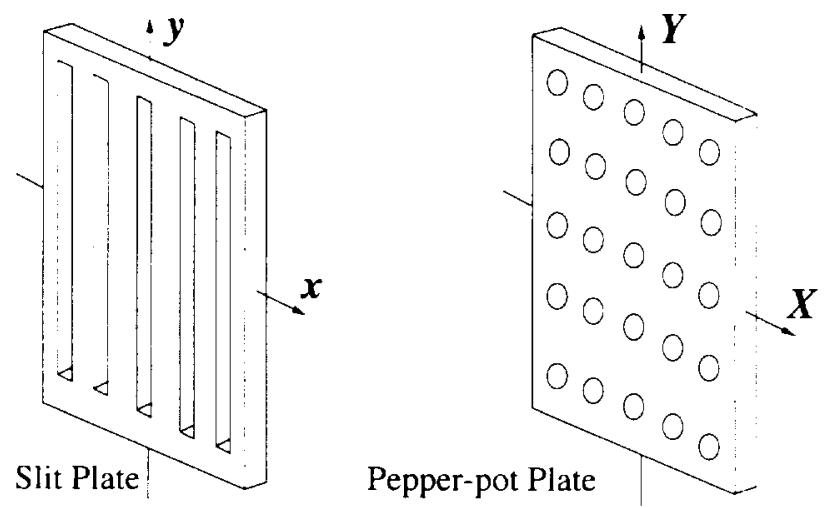

Figure 1: Beam masks in emittance measurement: slit plate (left) and pepper-pot plate (right)

$\mathrm{L}$ is the distance between the slit plate (or pepper-pot) and the phosphor screen. For a low emittance beam, in order to have a high resolution, we need a big L. But a big L means a large space-charge emittance dilution to the beam. As known. any phosphor screen has a certain spot resolution limit, typically $\Delta=100 \mu \mathrm{m}$. L is then roughly determined by the incident beam's geometrical divergence $x^{\prime}$ with $L \geq \frac{\Delta p}{2 x} . p$ is the number of slits.

\footnotetext{
${ }^{1}$ On leave from Deutsches Elektronen-Synchrotron (DESY) -MPY- Notkestrasse 85, 22603 Hamburg. Germany
} 


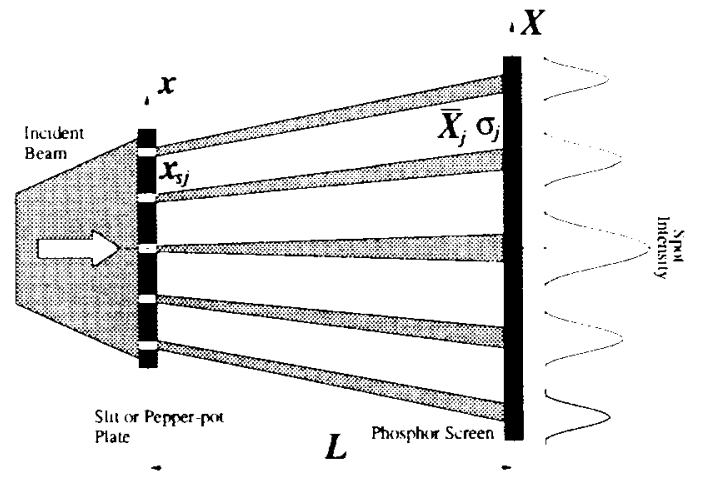

Figure 2: Typical setup for emittance measurement: Incident beam comes from left. $x$ is the slit or pepper-pot plate coordinate and $X$ is that for the phosphor screen. Slits length runs perpendicularly to the paper.

For a fixed beam radius at the slit plate, the beam divergence is given by $x^{\prime}=\frac{x_{n}^{\prime}}{\gamma}$, with $x_{n}^{\prime}$ being normalized beam divergence, and $\gamma$ the Lorentz factor. $x_{n}^{\prime}$ is a constant for different $\gamma$. So for a given phosphor screen resolution, lower energy beam is more favorable for a fixed L. But as known, low energy means a large space-charge emittance dilution, increasing the measurement errors.

The intensity of beamlet spots on a phosphor screen is primarily proportional to the number of particles in the beamlets which are hitting the screen.

We use two coordinates to locate the beamlets: one for the slits $(x)$, the other for the screen $(X)$. Their origins do not need to be aligned. But they are assumed to have the same unit. It is also assumed that the size of slits is all the same and is very small compared with the beam size. So we can use the slit's center line as its location $\left(x_{i}\right)$. L is small enough so that there is no overlap between beamlet spots on the screen. Our goal is to find an emittance formula $\left(\epsilon_{x}\right)$ which employs only geometrical parameters of the slits and the spots on screen. that is, slits position $\left(x_{s j}\right)$, mean position of spots on screen $\left(\bar{X}_{j}\right)$, and rms size of spots on screen $\left(\sigma_{j}\right)$.

It has to be made clear that a measured emittance is not beam's real mathematical emittance because of the following two reasons. Firstly, the measured emittance is based on a subset of particles instead of the whole beam. Secondly, there always exists space charge contribution to the final beamlets. So normally a measured emittance is at most an estimation of the real beam emittance.

\section{Derivation of Slit Emittance Formula}

Assume total number of particles before slit is $\mathrm{M}$, their transverse positions and momenta are

$$
\begin{aligned}
& \text { positions }\left(x_{i}, y_{i}\right) \quad i=1,2 \ldots . M \\
& \text { momenta }\left(x_{i}^{\prime}, y_{i}^{\prime}\right) \text {. }
\end{aligned}
$$

$x_{i}^{\prime}$ is defined as (Fig. 3)

$$
x_{i}^{\prime} \equiv \frac{X_{i}-x_{2}}{L}
$$


We only discuss $\mathrm{x}$ plane emittance $\left(\epsilon_{x}\right)$ here. The $\epsilon_{y}$ is identical to the following derivation.

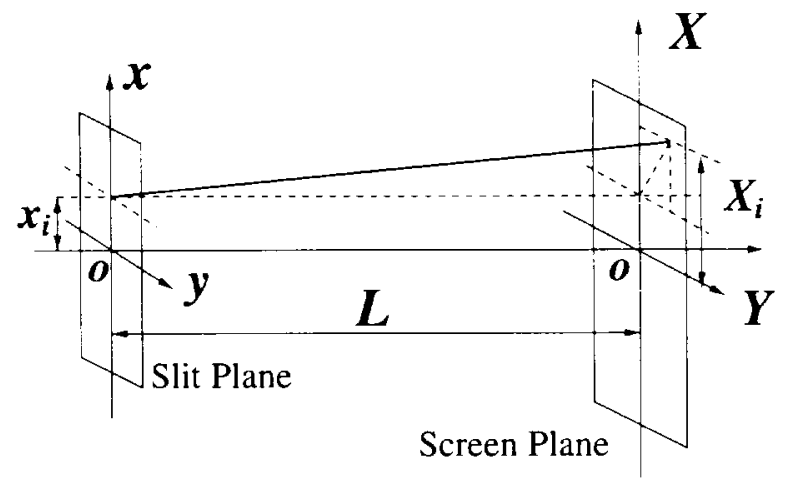

Figure 3: Illustration of $x_{i}^{\prime} . \mathrm{L}$ is the gap between the slit plate and the screen.

Total rms emittance is defined by

$$
\epsilon_{x} \equiv \sqrt{\left.<x^{2}><x^{\prime 2}>-<x x^{\prime}\right\rangle^{2}}
$$

with

$$
<x^{2}>\equiv \frac{1}{M} \sum_{i=1}^{M}\left(x_{i}-\bar{x}\right)^{2}, \quad \bar{x} \equiv \frac{1}{M} \sum_{i=1}^{M} x_{i}, \quad<x x^{\prime}>\equiv \frac{1}{M} \sum_{i=1}^{M}\left(x_{i}-\bar{x}\right)\left(x_{i}^{\prime}-\bar{x}^{\prime}\right) .
$$

Applying a mask to the beam, say, a slit plate, we have $X$ particles behind the slit. Clearly; $N \leq M$. This is a subset of the initial beam. Then the subset beam's rms emittance is given by Eqs. (3) and (4) with $\langle\bullet\rangle=\frac{1}{v} \sum_{i=1}^{N} \bullet$. From now on we will only discuss this subset emittance.

$2.1<x>$

$$
\begin{aligned}
<x> & =\bar{x}=\frac{1}{N} \sum_{i=1}^{N} x_{i} \\
& =\frac{1}{N}\left(\sum_{i=1}^{n_{1}} x_{1 i}+\sum_{i=1}^{n_{2}} x_{2 i}+\cdots+\sum_{i=1}^{n_{p}} x_{p i}\right) \\
& \approx \frac{1}{N}\left(n_{1} x_{s 1}+n_{2} x_{s 2}+\cdots+n_{p} x_{s p}\right)=\frac{1}{N} \sum_{j=1}^{p} n_{j} x_{s j} .
\end{aligned}
$$

$x_{s j}(j=1,2, \ldots, p)$ is $\mathrm{j}$-th slit position. $p$ is the number of slits. $n_{j}$ is number of particles through the $\mathrm{j}$-th slit, which is proportional to the spot intensity on screen. $x_{j i}(j=1,2, \cdots, p$ and $i=$ $\left.1,2, \cdots, n_{j}\right)$ are positions of particles passing through $\mathrm{j}$-th slit. The whole set of $\left\{x_{j i}\right\}$ is exactly equal to $\left\{x_{i} \mid i=1,2, \cdots, N\right\}$. This grouping scheme is also made for spot positions $\left\{X_{j i}\right\}$ and beam divergences $\left\{x_{j i}^{\prime}\right\}$ in the following discussions. Clearly.

$$
n_{1}+n_{2}+\cdots+n_{p}=N \text {. }
$$


$2.2<x^{\prime}>$

$$
\begin{aligned}
<x^{\prime}>= & \bar{x}^{\prime}=\frac{1}{N} \sum_{i=1}^{N} x_{i}^{\prime} \stackrel{E q .(2)}{=} \frac{1}{N} \sum_{i=1}^{N} \frac{X_{i}-x_{i}}{L} \\
\approx & \frac{1}{N L}\left(\sum_{i=1}^{n_{1}} X_{1 i}-n_{1} x_{s 1}+\sum_{i=1}^{n_{2}} X_{2 i}-n_{2} x_{s 2}+\cdots+\sum_{i=1}^{n_{p}} X_{p i}-n_{p} x_{s p}\right) \\
= & \frac{1}{N L}\left[n_{1}\left(\frac{1}{n_{1}} \sum_{i=1}^{n_{1}} X_{1 i}-x_{s 1}\right)+n_{2}\left(\frac{1}{n_{2}} \sum_{i=1}^{n_{2}} X_{2 i}-x_{s 2}\right)+\cdots+\right. \\
& \left.n_{p}\left(\frac{1}{n_{p}} \sum_{i=1}^{n_{p}} X_{p i}-x_{s p}\right)\right] .
\end{aligned}
$$

Define

$$
\bar{x}_{j}^{\prime} \equiv \frac{\frac{1}{n_{j}} \sum_{i=1}^{n_{3}} X_{j i}-x_{s j}}{L}
$$

and

$$
\bar{X}_{j} \equiv \frac{1}{n_{j}} \sum_{i=1}^{n_{j}} X_{j i}
$$

The latter is the mean position of $\mathrm{j}$-th spot on the screen. The former can be rewritten as

$$
\bar{x}_{j}^{\prime}=\frac{\bar{X}_{j}-x_{s j}}{L}
$$

using Eq. (9). Then we have

$$
<x^{\prime}>=\frac{1}{N}\left[n_{1} \overline{x_{1}^{\prime}}+n_{2} \overline{x_{2}^{\prime}}+\cdots+n_{p} \overline{x_{p}^{\prime}}\right]=\frac{1}{N} \sum_{j=1}^{p} n_{j} \overline{x_{j}^{\prime}} .
$$

We can redo Eq. (11) by directly using $x_{i}^{\prime}$.

$$
\begin{aligned}
\left.<x^{\prime}\right\rangle & =\frac{1}{N} \sum_{i=1}^{N} x_{i}^{\prime} \\
& =\frac{1}{N}\left(\sum_{i=1}^{n_{1}} x_{1 i}^{\prime}+\sum_{i=1}^{n_{2}} x_{2 i}^{\prime}+\cdots+\sum_{i=1}^{n_{p}} x_{p i}^{\prime}\right) \\
& =\frac{1}{N}\left[n_{1}\left(\frac{1}{n_{1}} \sum_{i=1}^{n_{1}} \overline{x_{1 i}^{\prime}}\right)+n_{2}\left(\frac{1}{n_{2}} \sum_{i=1}^{n_{2}} \overline{x_{2 i}^{\prime}}\right)+\cdots+n_{p}\left(\frac{1}{n_{p}} \sum_{i=1}^{n_{p}} x_{p i}^{\prime}\right)\right] \\
& \stackrel{\text { Eq. (11) }}{=} \frac{1}{N}\left[n_{1} \overline{x_{1}^{\prime}}+n_{2} \overline{x_{2}^{\prime}}+\cdots+n_{p} \overline{x_{p}^{\prime}}\right] .
\end{aligned}
$$

It can be easily found that

$$
\overline{x_{j}^{\prime}}=\frac{1}{n_{j}} \sum_{i=1}^{n_{j}} x_{j i}^{\prime}
$$

From the righthand side, we know that $\bar{x}_{j}^{\prime}$ is just the mean divergence of particles passing through the $\mathrm{j}$-th slit. Equation (10) provides a way to evaluate it by using the beamlet spot information. 
If the origins of the two coordinates $x$ and $X$ do not coincide. say. $X=x+a$, we have

$$
x_{j 2}^{\bar{\prime}}=\frac{\bar{X}_{j}+a-x_{s j}}{L}=\frac{\bar{X}_{j}-x_{s j}}{L}+\frac{a}{L}=\bar{x}_{j}^{\prime}+\frac{a}{L} .
$$

This means that for each slit spot. its mean divergence is dependent on the coordinate alignment. But it will be demonstrated below that this does not affect the emittance as a whole.

The above alignment error is also true for the overall mean divergence of the beamlets:

$$
\overline{x_{a}^{\prime}}=\frac{1}{N} \sum_{j=1}^{p} n_{j} x_{j a}^{\prime}=\frac{1}{N}\left(\sum_{j=1}^{p} n_{j} \overline{x_{j}^{\prime}}+\sum_{j=1}^{p} n_{j} \frac{a}{L}\right) \stackrel{E_{q .}(6)}{=} \bar{x}^{\prime}+\frac{a}{L} .
$$

$2.3<x^{2}>$

$$
\begin{aligned}
<x^{2}> & =\frac{1}{N} \sum_{i=1}^{N}\left(x_{i}-\bar{x}\right)^{2} \\
& =\frac{1}{N}\left[\sum_{i=1}^{n_{1}}\left(x_{1 i}-\bar{x}\right)^{2}+\sum_{i=1}^{n_{2}}\left(x_{2 i}-\bar{x}\right)^{2}+\cdots+\sum_{i=1}^{n_{P}}\left(x_{p i}-\bar{x}\right)^{2}\right] \\
& \approx \frac{1}{N} \sum_{j=1}^{p} n_{j}\left(x_{s j}-\bar{x}\right)^{2}
\end{aligned}
$$

$2.4<x^{\prime 2}>$

$$
\begin{aligned}
<x^{\prime 2}>= & \frac{1}{N}\left\{\sum_{i=1}^{N}\left(\bar{x}_{i}^{\prime}-\overline{x^{\prime}}\right)^{2}\right\} \\
= & \frac{1}{N}\left\{\sum_{i=1}^{n_{1}}\left[\left(x_{1 i}^{\prime}-\overline{x_{1}^{\prime}}\right)+\left(\overline{x_{1}^{\prime}}-\overline{x^{\prime}}\right)\right]^{2}+\sum_{i=1}^{n_{2}}\left[\left(\overline{x_{2 i}^{\prime}}-\overline{x_{2}^{\prime}}\right)+\left(\overline{x_{2}^{\prime}}-\overline{x^{\prime}}\right)\right]^{2}+\cdots+\right. \\
& \left.\sum_{i=1}^{n_{p}}\left[\left(x_{p i}^{\prime}-\overline{x_{p}^{\prime}}\right)+\left(\overline{x_{p}^{\prime}}-\overline{x^{\prime}}\right)\right]^{2}\right\} \\
= & \frac{1}{N}\left\{\sum_{i=1}^{n_{1}}\left(\bar{x}_{1 i}^{\prime}-\overline{x_{1}^{\prime}}\right)^{2}+2 \sum_{i=1}^{n_{1}}\left(\bar{x}_{1 i}^{\prime}-\overline{x_{1}^{\prime}}\right)\left(\overline{x_{1}^{\prime}}-\overline{x^{\prime}}\right)+\sum_{i=1}^{n_{1}}\left(\overline{x_{1}^{\prime}}-\overline{x^{\prime}}\right)^{2}+\right. \\
& \sum_{i=1}^{n_{2}}\left(\bar{x}_{2 i}^{\prime}-\overline{x_{2}^{\prime}}\right)^{2}+2 \sum_{i=1}^{n_{2}}\left(\overline{x_{2 i}^{\prime}}-\overline{x_{2}^{\prime}}\right)\left(\overline{x_{2}^{\prime}}-\overline{x^{\prime}}\right)+\sum_{i=1}^{n_{2}}\left(\overline{x_{2}^{\prime}}-\overline{x^{\prime}}\right)^{2}+\cdots+ \\
& \left.\sum_{i=1}^{n_{p}}\left(x_{p i}^{\prime}-\overline{x_{p}^{\prime}}\right)^{2}+2 \sum_{i=1}^{n_{p}}\left(\bar{x}_{p i}^{\prime}-\overline{x_{p}^{\prime}}\right)\left(\overline{x_{p}^{\prime}}-\overline{x^{\prime}}\right)+\sum_{i=1}^{n_{p}}\left(\overline{x_{p}^{\prime}}-\overline{x^{\prime}}\right)^{2}\right\}
\end{aligned}
$$

From Eq. (13), we get

$$
\sum_{i=1}^{n_{j}}\left(x_{j i}^{\prime}-\bar{x}_{j}^{\prime}\right) \equiv 0 \quad \forall j=1,2, \ldots, p .
$$


With this equation, it is known that all the middle terms vanish. Then we obtain

$$
\begin{aligned}
\left.<x^{\prime 2}\right\rangle= & \frac{1}{V}\left\{\sum_{i=1}^{n_{1}}\left(\bar{x}_{1 i}^{\prime}-\overline{x_{1}^{\prime}}\right)^{2}+n_{1}\left(\overline{x_{1}^{\prime}}-\overline{x^{\prime}}\right)^{2}+\right. \\
& \sum_{i=1}^{n_{2}}\left(\bar{x}_{2 i}^{\prime}-\overline{x_{2}^{\prime}}\right)^{2}+n_{2}\left(\overline{x_{2}^{\prime}}-\overline{x^{\prime}}\right)^{2}+\cdots+ \\
& \left.\sum_{i=1}^{n_{p}}\left(x_{p i}^{\prime}-\overline{x_{p}^{\prime}}\right)^{2}+n_{p}\left(\overline{x_{p}^{\prime}}-\overline{x^{\prime}}\right)^{2}\right\} .
\end{aligned}
$$

Define:

$$
\sigma_{x_{j}} \equiv \sqrt{\frac{1}{n_{j}} \sum_{i=1}^{n_{j}}\left(x_{j i}^{\prime}-\overline{x_{j}^{\prime}}\right)^{2}} .
$$

which is the rms divergence of beamlet at the $\mathrm{j}$-th slit. This value can be calculated by

$$
\sigma_{x_{j}^{\prime}}=\frac{\sigma_{j}}{L}
$$

with

$$
\sigma_{j} \equiv \sqrt{\frac{1}{n_{j}} \sum_{i=1}^{n_{j}}\left(X_{j i}-\bar{X}_{j}\right)^{2}} .
$$

which is the rms spot size of $j$-th beamlet on screen.

Equation (21) can be proofed as follows:

$$
\begin{aligned}
\sigma_{x^{\prime}}{ }^{2} & =\frac{1}{n_{j}} \sum_{i=1}^{n_{j}}\left(x_{j i}^{\prime}-\bar{x}_{j}^{\prime}\right)^{2} \\
E q s . & \stackrel{(2)}{=}(10) \frac{1}{n_{j}} \sum_{i=1}^{n_{j}}\left(\frac{X_{j i}-x_{s j}}{L}-\frac{\bar{X}_{j}-x_{s j}}{L}\right)^{2} \\
& =\frac{1}{n_{j}} \sum_{i=1}^{n_{j}}\left(\frac{X_{j i}-\bar{X}_{j}}{L}\right)^{2} \\
& =\frac{1}{n_{j} \sum_{i=1}^{n_{j}}\left(X_{j i}-\bar{X}_{j}\right)^{2}} \\
& \stackrel{L^{2}}{=}\left(\frac{\sigma_{j}}{L}\right)^{2} .
\end{aligned}
$$

With Eq. (20), Eq. (19) can be written as below

$$
<x^{\prime 2}>=\frac{1}{N} \sum_{j=1}^{p}\left[n_{j} \sigma_{x_{j}^{\prime}}^{2}+n_{j}\left(\bar{x}_{j}^{\prime}-\bar{x}^{\prime}\right)^{2}\right] .
$$

According to Eqs. (14) and (15), it is found that the value of $\left\langle x^{\prime 2}\right\rangle$ is independent of the alignment of $x$ and $X$ origins, since

$$
\bar{x}_{j a}^{\bar{\prime}}-\bar{x}_{a}^{\prime}=\bar{x}_{j}^{\prime}-\overline{x^{\prime}}
$$


$2.5<x x^{\prime}>$

$$
<x x^{\prime}>=\frac{1}{N} \sum_{i=1}^{N}\left(x_{i}-\bar{x}\right)\left(x_{i}^{\prime}-\dot{x}^{\prime}\right)
$$

Let us first discuss the alignment of origins. From Eq. (2), it is straightforward to get

$$
x_{i a}^{\prime}=\frac{X_{j i}+a-x_{s j}}{L}=\frac{X_{j i}-x_{s j}}{L}+\frac{a}{L}=x_{i}^{\prime}+\frac{a}{L} .
$$

In the above, it was assumed that the particle was from the $\mathrm{j}$-th slit. With Eq. (15), we know

$$
x_{i a}^{\prime}-\bar{x}_{a}^{\prime}=x_{i}^{\prime}-\bar{x}^{\prime} .
$$

This means that $\left\langle x x^{\prime}\right\rangle$ is independent of the alignment of the origins.

$$
\begin{aligned}
\left\langle x x^{\prime}\right\rangle \quad & =\frac{1}{N} \sum_{i=1}^{N}\left(x_{i} x_{i}^{\prime}-x_{i} \overline{x^{\prime}}-\bar{x} x_{i}^{\prime}+\bar{x} \overline{x^{\prime}}\right) \\
& =\frac{1}{N} \sum_{i=1}^{N} x_{i} x_{i}^{\prime}-\bar{x} \overline{x^{\prime}} \\
& \approx \frac{1}{N}\left(\sum_{i=1}^{n_{1}} x_{s 1} x_{1 i}^{\prime}+\sum_{i=1}^{n_{2}} x_{s 2} x_{2 i}^{\prime}+\cdots+\sum_{i=1}^{n_{p j}} x_{s p} x_{p i}^{\prime}-N \bar{x} \overline{x^{\prime}}\right) \\
& \stackrel{E q .(13)}{=} \frac{1}{N}\left(n_{1} x_{s 1} \overline{x_{1}^{\prime}}+n_{2} x_{s 2} \overline{x_{2}^{\prime}}+\cdots+n_{p} x_{s p} \overline{x_{p}^{\prime}}-N \bar{x} \overline{x^{\prime}}\right) \\
& =\frac{1}{N}\left(\sum_{j=1}^{p} n_{j} x_{s j} \overline{x_{j}^{\prime}}-N \ddot{x} \overline{x^{\prime}}\right)
\end{aligned}
$$

\section{$2.6 \epsilon_{x}^{2}$}

With Eq. (16) for $\left\langle x^{2}>\right.$. Eq. (24) for $\left\langle x^{\prime 2}>\right.$. and Eq. (29) for $\left\langle x x^{\prime}\right\rangle^{2}$, we obtain

$$
\begin{aligned}
\epsilon_{x}^{2} & =\left\langle x^{2}\right\rangle\left\langle x^{\prime 2}>-<x x^{\prime}\right\rangle^{2} \\
& \approx \frac{1}{N^{2}}\left\{\left[\sum_{j=1}^{p} n_{j}\left(x_{s j}-\bar{x}\right)^{2}\right]\left[\sum_{j=1}^{p}\left[n_{j} \sigma_{x_{j}^{\prime}}{ }^{2}+n_{j}\left(\overline{x_{j}^{\prime}}-\overline{x^{\prime}}\right)^{2}\right]\right]-\left[\sum_{j=1}^{p} n_{j} x_{s j} \overline{x_{j}^{\prime}}-N \bar{x} \overline{x^{\prime}}\right]^{2}\right\} .
\end{aligned}
$$

This is the final slit emittance formula. All the terms in the formula are expressed by slit positions and beamlet spots parameters on the screen. Specifically, they are

- $x_{s j}-\mathrm{j}$-th slit's position;

- $p$ - total number of slits:

- $n_{j}$ - number of particles passing through $\mathrm{j}$-th slit and hitting the screen. Practically it is a weighting of spot intensity: 
- $\bar{x}$ - mean position of all beamlets (Eq. (5));

- $\overline{x_{j}^{\prime}}-$ mean divergence of $\mathrm{j}$-th beamlet (Eq. (10)):

- $\overline{x^{\prime}}$ - mean divergence of all beamlets (Eq. (11)):

- $\sigma_{x_{j}^{\prime}}-$ rms divergence of $\mathrm{j}$-th beamlet (Eq. (21)).

\subsection{Generalization of Slit Emittance to Pepper-pot}

The generalization of the above slit emittance formula to the pepper-pot case is straightforward. Instead of projecting slit image lines to the $\mathrm{x}$ axis, we sum up all pepper-pot spot images in $y$ direction for $\epsilon_{x}$ and in $\mathrm{x}$ direction for $\epsilon_{y}$ (Fig. 4).

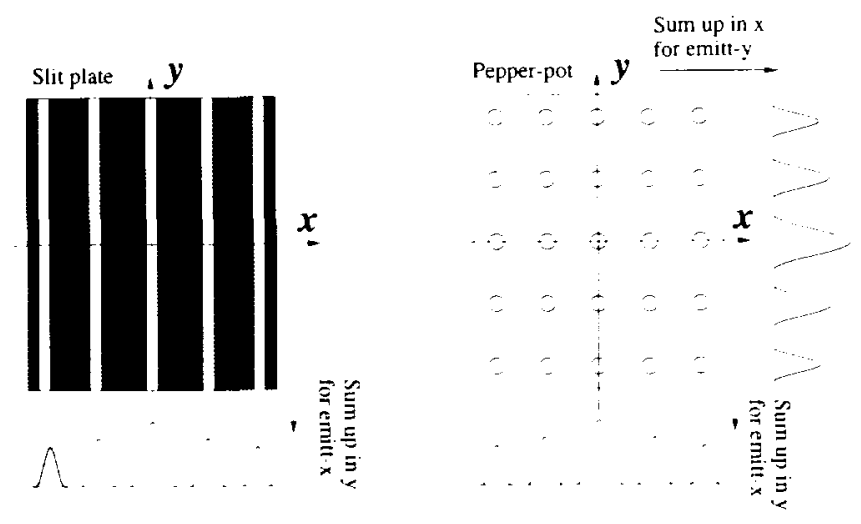

Figure 4: Illustration for slit and pepper-pot image analysis

\section{Closing Remarks}

There is a general question that what is the relationship between beam's real mathematical emittance and that measured using either slits or pepper-pot, i.e. between total emittance and subset emittance. If this question can be addressed, we are then in a position to give an estimation of measurement er rors between $\epsilon$ and $\epsilon_{\text {subset }}$ and the difference between $\epsilon_{\text {slits }}$ and $\epsilon_{\text {pepper-pot }}$.

For a subset slit emittance measurement, the formula derived in this note is mathematically rigid. This formula applies only to rms emittances, not to ellipse emittances.

In a measurement setup, we do not need to align the slit plate coordinate with the screen's. because all the values in the formula are relative to their respective coordinates only.

\section{References}

[1] "A VUV free electron laser at the TESLA test facility at DESY. Conceptual design report". DESY Print. June 1995. TESLA-FEL 9.5-03 